\title{
Présentation du GF2D
}

\author{
Afiwa Kafui Kuwonu \\ Generalsekretärin Groupe de réflexion et d'action de Femme, Dé- \\ mocratie et Développement (GF2D/CRIFF), Lomé, Togo
}

Le Togo est un pays d'Afrique de l'Ouest ayant des frontières communes avec le Bénin à l'est, le Burkina Faso au nord, et le Ghana à l'ouest. Sa façade sud est ouverte sur le golfe de Guinée. Il est organisé en collectivités territoriales à savoir 5 régions administratives réparties en 33 préfectures et sous préfectures.

La population féminine représente plus de $51 \%$ de la population totale estimée à 5.598.000 en 2008. Le peuple togolais est constitué d'une cinquantaine d'ethnies.

L'histoire enseigne que le Togo a des liens historiques très forts avec l'Allemagne. En effet en 1884, le roi MLAPA III de Togoville, a signé un traité de protectorat avec l'Allemagne représenté par Gustav Nachtigal. Cette colonisation allemande a duré jusqu'à la fin de la première Guerre mondiale. La colonie est ensuite divisée entre le Royaume Unie et la France. La partie française est le Togo d'aujourd'hui.

Le Togo a accédé à l'indépendance en 1960 et a connu de 1967 à 1990 le monopartisme.

C'est à partir des années 1990 que le Togo à l'instar de beaucoup de pays africains, a amorcé sa démocratisation. C'est dans cette mouvance démocratique que les GF2D a été créé en 1992 par une trentaine de femmes d'horizons socio professionnels divers. Ces femmes ont produit dans un premier temps en 1994 le "Manifeste Autrement". Ce document après avoir fait le constat de l'énorme potentiel que constitue la femme togolaise invite à penser autrement son rôle dans la nouvelle société démocratique.

A partir de ce manifeste, les premiers axes d'intervention du GF2D ont été identifiés. Entend qu'association née du renouveau démocratique, le GF2D apporte sa contribution au changement en orientant ses réflexions et actions vers les voies et moyens pouvant permettre à la femme de saisir l'occasion offerte par le contexte démocratique pour contribuer au développement du pays.

L'association s'est dotée d'une structure technique appelée le Centre de Recherche, d'Information et de Formation pour la Femme (CRIFF) qui se charge de l'exécution de ses programmes contenus dans un plan revu tous les 5 ans.

L'éducation aux droits des femmes et aux droits de l'homme en général, la recherche et la publication ainsi que des actions de plaidoyer et lobbying sont les approches utilisées par le GF2D pour mettre en œuvre sa mission. Trois programmes servent de cadre pour la mise en œuvre de sa mission et sont mis en œuvre depuis 1994. Il s'agit de:

- Programme des Services Juridiques

- Programme d'Education Civique

- Programme socio économique
Dans le cadre de cette présentation, l'accent sera mis sur le programme des services juridiques en direction des femme d'une part et sur les grandes actions du GF2D visant à promouvoir la démocratie et de l'Etat de droit d'autre part.

\section{Les Services Juridiques en Direction des Femmes}

La formation des parajuristes est le maillon essentiel des actions du GF2D.

\section{La formation des parajuristes}

„Nul n'est sensé ignorer la loi“ dit l'adage latin. Mais cette maxime ne pourrait être objectivement appliquée aux africains en général et aux togolais en particulier. Car la majorité de la population ne sait ni lire ni écrire. En l'occurrence 60,4\% de femmes sont analphabètes. Or un enracinement de la démocratie ne peut se faire sans la prise en compte des droits des femmes et ces dernières ne peuvent jouer pleinement leur rôle si elles ne connaissent ce qui est reconnu comme droit. Le GF2D a compris cette nécessité et fait connaître aux populations surtout les femmes du milieu urbain comme du milieu rural les lois qui les régissent et qui protègent leurs droits. Il a alors conçu le programme de formation des parajuristes par le truchement duquel l'information juridique est transmise aux populations dans un langage simplifié et facile à la compréhension et parfois dans les langues locales.

Les parajuristes sont des femmes et quelques hommes dynamiques, dévoués à leur communauté et mus par la volonté de contribuer à faire connaître les droits des femmes, à faire régner l'équité entre les citoyens. Ils sont de toutes les ethnies, de tous les bords politiques et exercent divers métiers. Ils sont cultivateurs, commerçantes, matrones, sage-femme, enseignants, fonctionnaires, journalistes, artisanes et gardiens des us et coutumes.

Les parajuristes ne sont pas des spécialistes du droit mais sont formés de manière pratique en droit de la famille, droit successoral, droit immobilier, en droit social, économique et politique, en techniques de communication et de mediation.

Après 15 ans de formation, le GF2D dispose à ce jour d'environ 600 parajuristes répartis en 33 réseaux et dont les activités couvrent les 33 préfectures et sous préfectures du Togo.

Les activités des parajuristes sont de trois ordres.

\section{La sensibilisation}

La tâche primordiale des parajuristes est de combattre l'analphabétisme juridique. Ils ont systématisé la vulgarisation du Code des personnes et de la famille par différentes approches: causerie-débat, sketch, théatre.

Certains parajuristes ont reçu une formation complémentaire et sont devenus des points focaux „Santé de reproduction" et VIH SIDA et des points focaux droits successoraux. 


\section{La médiation}

Lorsque les mésententes naissent entre les membres des communautés, les parajuristes sont appelés à jouer le rôle de médiation. Ce mode de règlement est très apprécié par les populations comme en témoigne une parajuriste de la région maritime: “j’ai réussi à réconcilier 5 ménages en instance de divorce par la médiation. J'ai constaté suite à ces médiations que les hommes aussi manquent d'information juridique.“

\section{L'assistance juridique}

En somme les parajuristes constituent auprès de la population à la base des relais du GF2D pour transmettre l'information juridique et assister les femmes à travers les centres d'écoute.

\section{L'assistance juridique aux femmes à travers les centres d'écoute}

Le centre d'écoute et d'assistance juridique encore appelé clinique juridique fournit la consultation juridique. La consultation ou l'aide juridique est une activité ayant pour but d'offrir aux femmes et à toute la communauté des informations juridiques pouvant les aider dans le règlement des litiges familiaux, profesionnels, ou dans leurs rapports avec l'administration.

Le centre est dirigé par les juristes et les parajuristes.

Le GF2D dispose de 7 sept d'écoute dont le plus grand se trouve au CRIFF au siège du GF2D. Ce dernier reçoit en moyenne 1400 cas par an. Le centre accueille gratuitement toute personne adulte ou enfant des deux sexes, de toute condition, de tout âge, de toute ethnie, de toute appartenance politique ou religieuse.

Le mode de règlement privilégié est le règlement à l'amiable afin de maintenir et préserver l'unité et l'harmonie dans la famille et au-delà dans la communauté. L'échec ou le refus de respecter l'arrangement résultant de la médiation ou la gravité de certaines infractions donnent la possibilité aux plaignants à saisir le tribunal. Dans ce cas, l'animatrice du centre apporte son assistance en indiquant le service juridique approprié, en aidant à rédiger une plainte ou une requête selon la nature du problème.

S'agissant des problèmes traités au centre d'écoute, ils sont relatifs à la famille (filiation, autorité parentale, succession, marriage ...) à l'état civil, aux violences, aux rapports professionnels (rupture du contrat de travail, protection sociale ...), et aux rapports des administrés avec l'administration.

En 2006 et en 2007 le centre de Lomé a reçu respectivement reçu 894 et 653 cas. En 20081436 cas dont 672 nouveaux cas ont été enregistrés.

\begin{tabular}{l|c|c|r}
\hline \multirow{2}{*}{ Mois } & \multicolumn{3}{|c}{ Nombre de cas reçus } \\
\cline { 2 - 4 } & Nouveaux cas & Anciens cas & Total \\
\hline Janvier & 55 & 57 & 112 \\
\hline Février & 35 & 37 & 72 \\
\hline Mars & 56 & 49 & 105 \\
\hline Avril & 69 & 57 & 126 \\
\hline Mai & 62 & 62 & 124 \\
\hline Juin & 41 & 64 & 105 \\
\hline Juillet & 56 & 56 & 112 \\
\hline Août & 54 & 71 & 125 \\
\hline Septembre & 54 & 98 & 152 \\
\hline Octobre & 81 & 84 & 165 \\
\hline Novembre & 66 & 72 & 138 \\
\hline Decembre & 43 & 57 & 100 \\
\hline Total & 672 & 764 & 1436 \\
\hline
\end{tabular}

$\triangle$ Motifs des Consultations des nouveaux cas

Des 672 nouveaux cas reçus au cours l'année 2008, 553 ont favorablement aboutis, soit $82,29 \%$. Des 764 anciens cas traités au cours de l'année 2008; 651 cas ont aboutis, soit un pourcentage de $85,20 \%$.

Au total 1204 des 1436 cas reçus ont favorablement abouti au cours de l'année 2008, soit 83,84\%.

En ce qui concerne particulièrement la succession, il se pose un réel problème d'articulation entre le droit moderne et le droit coutumier. Bien que la loi ne reconnaisse que le mariage célébré devant l'officier de l'état civil, le code des personnes et de la famille prévoit que la succession peut être réglée suivant la pratique coutumière. Or le système coutumier est patriarcal et ne confère pas les mêmes droits aux femmes qu' aux hommes surtout en ce concerne la terre.

La discrimination subie par la femme se manifeste de manière particulièrement violente lorsqu'elle est en situation de veuve qui au regard de la loi est en principe héritière des biens de son mari défunt. Le témoignage suivant d'une plaignante reçue au centre d'écoute permet d'apprécier concrètement ce problème „Mon époux est décédé à l'hôpital un mardi matin à dix heures. J'étais à son chevet avec une de mes bellessœurs lorsqu'il mourut. Nous fîmes les formalités nécessaires et le déposâmes à la morgue. Je rentrai à la maison attendant ma belle famille pour les dispositions à prendre en vue de l'enterrement. Deux beaux frères et ma belle sœur arrivent à quinze heures à bord d'une camionnette. D'entrée de jeu, ils me dirent qu'à partir de ce jour, le frère cadet de mon mari est désigné en attendant le conseil de famille, tuteur de mes enfants pour prendre soin de ces derniers. Aussi pour les questions relatives à la gestion de la maison je devais me référer à

\begin{tabular}{l|l|l|l|l|l|l|l|l}
\hline Mois & \multicolumn{7}{|c}{ Motifs } \\
\cline { 2 - 9 } & Succession & Violences & $\begin{array}{c}\text { Pension ali- } \\
\text { mentaire } \\
\text { d'enfants }\end{array}$ & $\begin{array}{c}\text { Garde } \\
\text { Autorité pa- } \\
\text { rentale }\end{array}$ & $\begin{array}{c}\text { Conflits } \\
\text { conjugaux }\end{array}$ & $\begin{array}{c}\text { Conflits } \\
\text { socio- com- } \\
\text { merciaux }\end{array}$ & $\begin{array}{c}\text { Pénal } \\
\text { Total }\end{array}$ \\
\hline Total & 59 & 122 & 160 & 61 & 10 & 179 & 33 & 48 \\
\hline & 8,77 & 18,15 & 23,82 & 9,07 & 1,48 & 26,63 & 4,91 \\
\hline
\end{tabular}

Nombre de cas résolus avec l'assistance du centre 
lui. Ils réclamèrent ensuite les clés de l'armoire où mon époux garde ses biens, ils réclamèrent également la clé de la voiture et de son bureau. Au moment de leur départ, ils emportèrent le réfrigérateur neuf qui venait d'être acheté. Ils prirent également le poste téléviseur et ses accessoires“.

Voilà la situation que subissent plusieurs femmes togolaises au lendemain du décès de leur mari.

Par ailleurs la souffrance que connaît la femme après le décès de son mari concerne aussi les rites de veuvage. Certains rites tels que le lévirat constituent également des graves à la dignité de la femme. Le GF2D mène des actions dans ce sens à travers un projet intitulé „Cinq ans pour réduire les violences liées aux rites de veuvages".

Vous convenez chères collègues qu'il y a un énorme travail de sensibilisation à faire pour sortir la femme de l'ignorance juridique et l'amener à jouir pleinement des droits reconnus par les instruments juridiques internationaux, régionaux et nationaux.

Mais l'éducation et l'assistance juridiques ne suffisant par pour faire des femmes de véritables actrices du processus démocratique au Togo, le GF2D mène des actions complémentaires pour créer les conditions propices à l'implication des femmes togolaises au processus de démocratisation et développement.

\section{Les Actions du GF2D en vue de Renforcement de la Démocra- tie et l'etat de droit}

\section{La promotion des femmes au leadership et à la participation à la vie politique et publique}

Le GF2D ne se contente pas de donner l'information juridique. Il renforce la capacité des femmes de sorte à assurer leur participation efficace aux processus de développement et de démocratisation de notre pays.

En effet dans le cadre de son deuxième programme, le GF2D mène un certain nombre d'activités d'éducation civique sur le terrain. Il s'agit de la formation des femmes à l'engagement à la vie publique; la formation des femmes membres des communautés à la base afin qu'elles participent à la prise de décision au niveau local et au niveau national.

Depuis 2004, le GF2D est fortement impliqué dans les différentes étapes du processus de règlement de la crise sociopolitique et de la consolidation de la démocratie au Togo. Il agit aux côtés des acteurs politiques pour l'enracinement de la démocratie. En effet depuis la prise par le Togo des 22 engagements vis-à-vis de l'Union Européenne, le GF2D participe en tant que représentante de la société civile au dialogue inter togolais qui a abouti à, la signature le 20 août 2006 d'un accord politique global. C'est sur la base de cette feuille de route que les élections législatives de 2007 ont été organisées. Dans le cadre desdites élections, le GF2D a non seulement sensibilisé la population sur le code électoral et les normes internationales régissant le vote mais aussi et surtout formé les femmes candidates à ces élections appartenant à divers partis politiques.
Par ailleurs dans la perspective des élections locales, le GF2D a initié des formations à l'endroit des femmes candidates potentielles en vue de bien outiller ces dernières pour s'imposer devant leurs concurrents hommes et remporter en nombre important les postes de conseillers.

Toujours dans le cadre des élections, le GF2D forme généralement les animateurs et animatrices d'éducation civique par le biais duquel il assure la vulgarisation des dispositions du code électoral et les principes universels régissant le vote.

En outre le GF2D met en œuvre un projet de promotion de femmes leaders en milieu rural à travers des formations de femmes et des membres des bureaux de CVD/CDQ (Comités Villageois de Développement et Comités de Développement de Quartier) sur des thèmes tels que: l'organisation, le rôle et le fonctionnement des CVD/CDQ, la prise de décision des femmes au sein des ménages et des communautés de base, la négociation, les techniques de communication, la recherche de financement, des pistes de réflexion sur la résolution des conflits au sein des CVD/CDQ, les enjeux de la décentralisation, etc.

Le GF2D accompagne les formations d'autres actions à l'endroit des décideurs pour amener ceux-ci à prendre des mesures concrètes favorables à l'implication des femmes dans les instances de gouvernance.

\section{Les actions de plaidoyer et lobbying}

Le GF2D interpelle constamment les décideurs politiques sur la situation des femmes et fait le plaidoyer pour en vue de son amélioration.

Partie prenante à l'Accord Politique Global, le GF2D à œuvré de sorte les parties prenantes au dialogue s'engagent à œuvrer en vue d'assurer une représentation équitable dans les processus électoraux dans la vie politique nationale. Ce qui a amené le gouvernement a adopté lors des élections législatives des mesures incitatives jugées toutefois pas suffisantes.

De même lors du renouvellement de la cour constitutionnelle une exigence de l'APG, le GF2D a mobilisé d'autres organisations de droits de femmes pour un faire un plaidoyer demandant la nomination de femmes parmi les juges constitutionnels. Ainsi pour la première fois dans l'histoire du Togo une femme siège à cette.

Le GF2D fait parti du réseau Coalition, Bonne gouvernance constitué dans le cadre d'un projet intitulé „Femme et Bonne gouvernance“ initié par le WiLDAF (Women in Law and Developement). Par une synergie d'action les organisations de la coalition ont adressé des recommandations aux décideurs politiques. Elles ont notamment demandé la gratuité de la césarienne et celle de l'école primaire. Ceci est effectif depuis la rentrée 2008-2009.

Le GF2D conscient que le combat pour les droits des femmes ne peut d'être efficacement mené en travaillant seul, collabore avec d'autres associations au niveau national, régional et international.

C'est pourquoi il se réjouit profondément de ce partenariat qui commence avec „Deutscher Juristinnenbund“ à travers ce cadre d'échange. 